\title{
Black Aortic Valve: Incidental Finding of Alkaptonuria
}

\author{
Christina Putz ${ }^{1}$ Franz Josef Putz ${ }^{2} \quad$ Andreas Keyser $^{1} \quad$ Christof Schmid $^{1}$ \\ ${ }^{1}$ Klinik für Herz-, Thorax- und herznahe Gefäßchirurgie, \\ Universitätsklinikum Regensburg, Regensburg, Germany \\ ${ }^{2}$ Klinik für Nephrologie, Universitätsklinikum Regensburg, \\ Regensburg, Germany

\begin{abstract}
Address for correspondence Dr. med. Christina Putz, Klinik für Herz-, Thorax- und herznahe Gefäßchirurgie, Universitätsklinikum Regensburg, Franz-Josef-Strauss-Allee 11,
\end{abstract} \\ Regensburg 93053, Germany (e-mail: christina.putz@ukr.de).
}

Thorac Cardiovasc Surg Rep 2021;10:e39-e41.

\begin{abstract}
Keyword

- aortic valve

- heart valve

- cardiac

Background Alkaptonuria is a rare autosomal recessive genetic disorder of tyrosine metabolism, which results in accumulation of homogentisic acid in various tissues, including the cardiovascular system.

Case Description We report on a 64-year-old man with mixed aortic valve disease who underwent conventional aortic valve replacement. Intraoperative aortotomy revealed black pigmentation of the intima of the ascending aorta and the aortic valve was observed with thickened and calcified dark black leaflets. Histopathological diagnosis of ochronosis of the aortic valve was made.

Conclusion Despite several previous signs and symptoms, the diagnosis of alkaptonuria was not established until aortic valve replacement.
\end{abstract}

\section{Introduction}

Alkaptonuria is an autosomal recessive genetic disorder of tyrosine metabolism with an estimated incidence of $1: 250,000$ to $1: 1,000,000$. A mutation in chromosome 3q21-23 causes a deficiency in the homogentisic acid oxidase enzyme and results in accumulation of homogentisic acid in extracellular tissues, leading to pathologic pigmentation termed ochronosis. ${ }^{1}$

Cardiovascular involvement is a rare but serious complication of the disease. We report on a case of aortic valve ochronosis, unveiled during aortic valve replacement.

\section{Case Description}

A 64-year-old male patient with mixed aortic valve disease was admitted to our institution. The patient complained of exertional dyspnea. He was in New York Heart Association functional class III. A transthoracic echocardiogram revealed severe trileaflet aortic stenosis with a valve area of $0.85 \mathrm{~cm}^{2}$, a peak gradient of $89 \mathrm{~mm} \mathrm{Hg}$, and a mean gradient of $58 \mathrm{~mm} \mathrm{Hg}$. Left ventricular function was normal (ejection fraction $=61 \%$ ). Cardiac catheterization confirmed diagnosis and revealed normal coronary arteries. All routine laboratory tests were normal. His medical history included bilateral total knee replacements due to degenerative arthritis, right shoulder arthrosis, cataract operation on the left eye, and hypertension.

The patient underwent conventional aortic valve replacement surgery. After aortotomy, the first conspicuous finding was a black pigmentation of the intima of the ascending aorta down to the aortic root. The tricuspid aortic valve presented with thickened and nodular calcified dark black leaflets (-Fig. 1). The pigmentation was also involving the annulus. A $25 \mathrm{~mm}$ Perimount Pericardial Bioprosthesis (Edwards Lifesciences, Irvine, California, United States) was implanted in a routine manner.

Histopathological examination of the aortic valve tissue demonstrated nodular calcification and dark pigmented received

February 23, 2021

accepted

DOI https://doi.org/

10.1055/s-0041-1728721. ISSN $2194-7635$. (c) 2021. The Author(s).

This is an open access article published by Thieme under the terms of the Creative Commons Attribution-NonDerivative-NonCommercial-License, permitting copying and reproduction so long as the original work is given appropriate credit. Contents may not be used for commercial purposes, or adapted, remixed, transformed or built upon. (https://creativecommons.org/ licenses/by-nc-nd/4.0/)

Georg Thieme Verlag KG, Rüdigerstraße 14, 70469 Stuttgart, Germany 


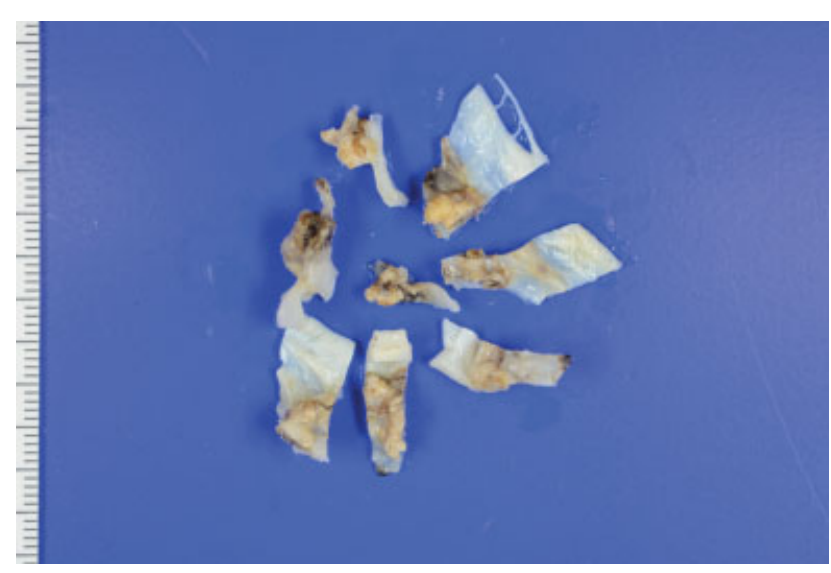

Fig. 1 Excised aortic valve leaflets showing ochronotic calcification.

deposits within the areas of calcification and focally in the noncalcified valvular tissue (-Fig. 2). A pathological diagnosis of ochronosis of the aortic valve was made.

The patient's postoperative course was uneventful and he was discharged from the hospital on postoperative day 9 .

A detailed history taken retrospectively after surgery revealed that the patient's urine turned dark if allowed to stand for several hours, which was known since childhood. The analysis of the patient's urine, which showed markedly raised levels of homogentisic acid at $1.3 \mathrm{~g} / \mathrm{L}$ (reference range: $<0.1 \mathrm{~g} / \mathrm{L})$, confirmed the diagnosis of alkaptonuria. Interestingly, it turned out that he is in urological treatment due to prostate stones.

During the prior joint replacement operations, black joint surfaces had been seen. On physical examination, brown pigmentation of the sclera of both eyes had been noticed (-Fig. 3). Furthermore, the cartilage of the outer ears showed gray discoloration (-Fig. 4).

Nevertheless, our patient had reached 64 years of age without being properly diagnosed with alkaptonuria despite the obvious symptoms of the disease. The diagnosis of ochronosis was not made until aortic valve replacement surgery.

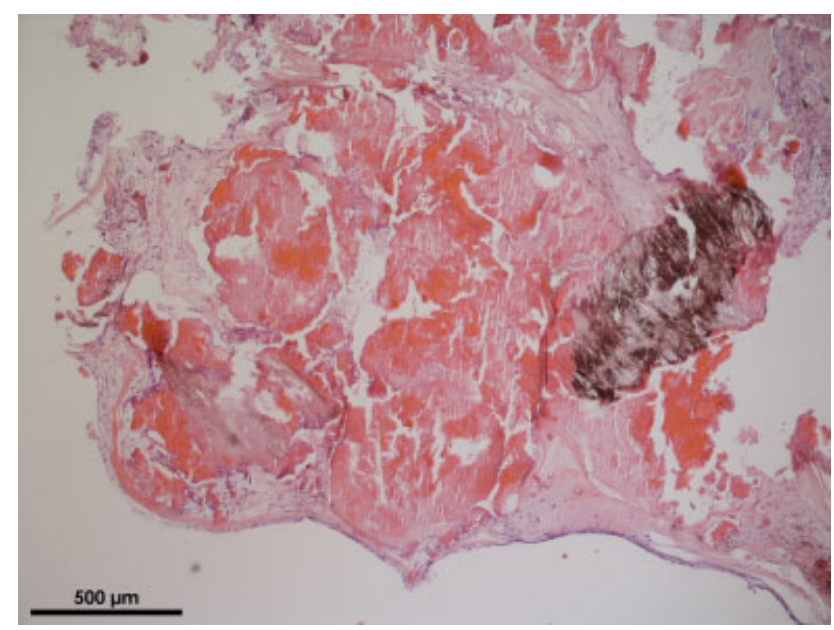

Fig. 2 Histopathological examination of a section of valvular leaflet showing ochronotic pigment deposition (hematoxylin and eosin, original $\times 5$ ) .

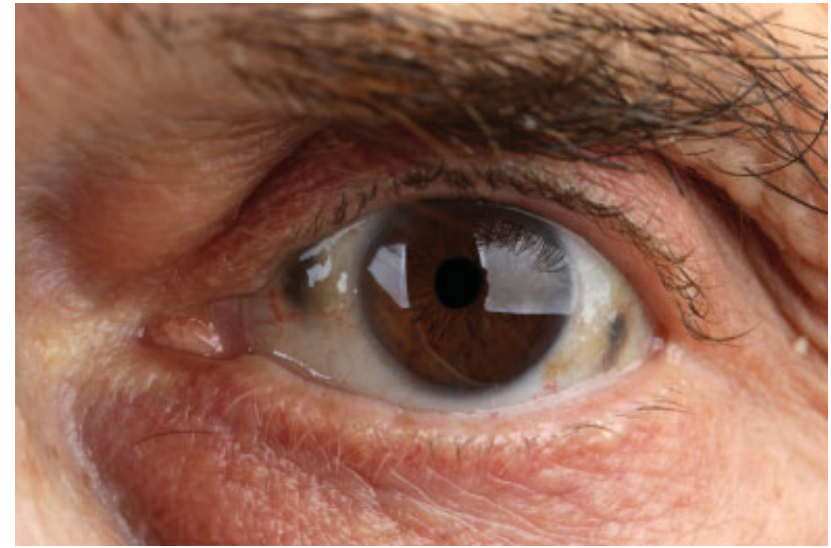

Fig. 3 Ochronotic pigmentation of the sclera of the eye.

\section{Discussion}

The clinical diagnosis of alkaptonuria depends on a classic triad of homogentisic aciduria, ochronosis, and degenerative arthropathy. The disease is usually diagnosed in childhood because of the darkened urine. In adulthood gray discoloration of the sclerae and the ear cartilage appears. As a result of homogentisic acid accumulation in hyaline cartilage, ochronotic arthropathy of large weight-bearing joints occurs

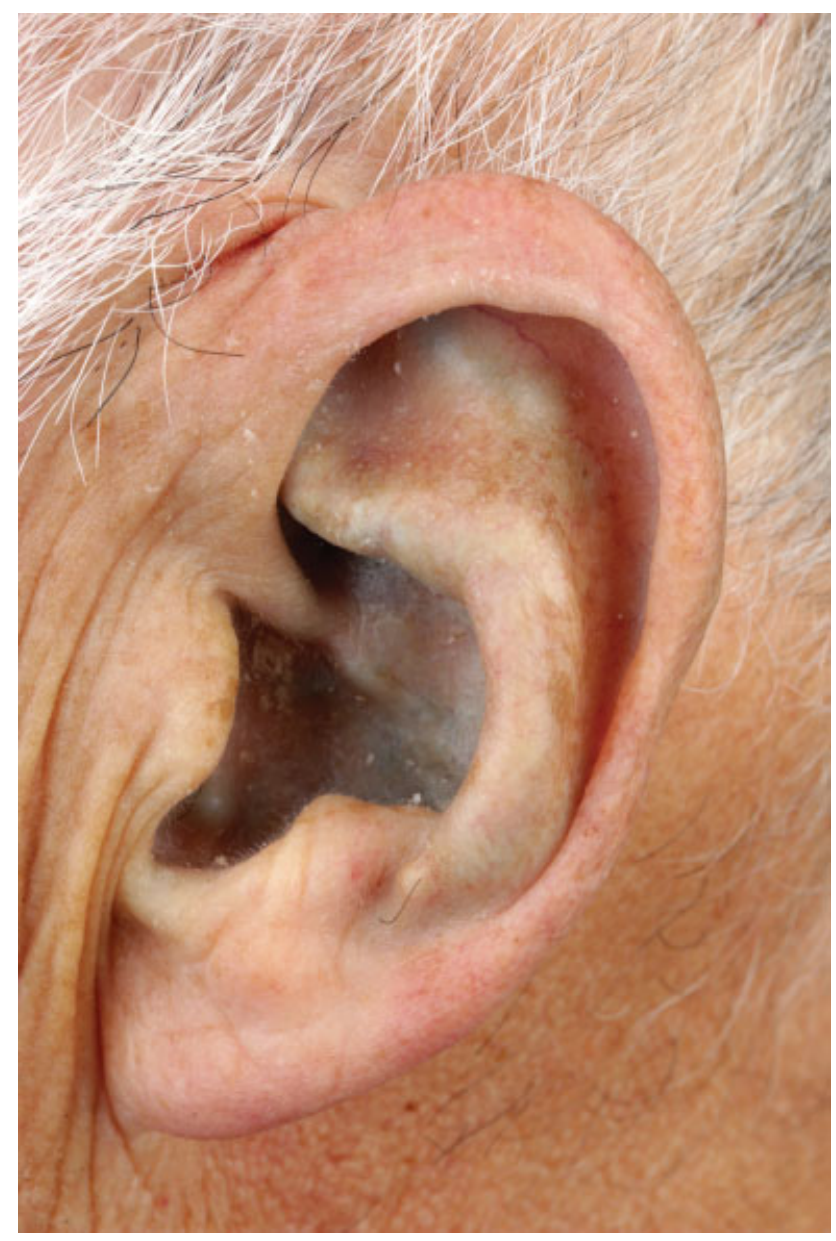

Fig. 4 Ochronotic pigmentation of the ear cartilage. 
in the fourth decade of life, often leading to joint replacement operations. Further clinical features affect the kidney, glands, and skin. Involvement of the cardiovascular system is a rare but serious complication. Depositions of ochronotic pigment have been found on cardiac valves, aortic intima, coronary arteries, endocardium, and pericardium.

Aortic valve stenosis is the most frequent cardiac manifestation of the disease. ${ }^{2}$ Ochronosis-associated valvular disease appears in the fifth decade of life due to the accumulation of pigment in valvular tissue. As a result of less pigmentation in the venous circulation compared with the arterial circulation, continuous mechanical stress and high pressure may also play an important role in the development of ochronosis. ${ }^{3}$ The ochronotic pigment in the aortic valve may stimulate dystrophic calcification and fibrosis, which leads to aortic valve stenosis. ${ }^{1}$

Currently there is no successful treatment of alkaptonuria, but the prognosis is relatively good. Therapy is generally directed at dealing with the complications of the disease and management of symptoms. Dietary restriction of protein supplemented with high dose of ascorbic acid is often recommended to reduce the amount of homogentisic acid in the system but does not significantly change the progression of the disease and is no long-term treatment for many patients. Alkaptonuria-associated arthritis is treated by physical therapy, nonsteroidal anti-inflammatory drugs, or total joint replacement.

Nitisinone, an inhibitor of 4-hydroxyphenylpyruvate dioxygenase, reduces the production and urinary excretion of homogentisic acid. Initial findings showed that nitisinone slowed down the clinical progression of alkaptonuria but long-term efficacy and side effects remain still unknown. ${ }^{4}$

Because these treatments have minimal effect on the disease process, ochronotic valvular disease leads to surgical valve replacement but has a good outcome after surgical intervention. The choice of valve prothesis in patients with ochronosis is still under debate. Further research will be needed to better understand the natural history of aortic valve ochronosis and the recurrence of the disease on heart valve prosthesis.

Although cardiac ochronosis is a very rare disease, cardiac surgeons should be aware of this condition since they might be confronted with the typical signs during elective cardiac surgery.

Conflict of Interest

None declared.

\section{References}

1 Selvakumar D, Sian K, Sugito S, Singh T. Ochronosis of the aortic valve. J Thorac Dis 2018;10(05):E332-E334

2 Lok ZS, Goldstein J, Smith JA. Alkaptonuria-associated aortic stenosis. J Card Surg 2013;28(04):417-420

3 Vo T, Edwards JRM. A black heart: aortic valve ochronosis secondary to alkaptonuria causing aortic stenosis. J Card Surg 2021;36 (02):758-760

4 Ranganath LR, Khedr M, Milan AM, et al. Nitisinone arrests ochronosis and decreases rate of progression of alkaptonuria: evaluation of the effect of nitisinone in the United Kingdom National Alkaptonuria Centre. Mol Genet Metab 2018;125 (1-2):127-134 